\title{
Pathogenetic Mechanisms of Relationship of Metabolic and Morphofunctional Disorders of Thyroid and Adrenal Glands in Diabetes Mellitus and Obesity
}

Saule Zhautikova(D), Khamida Abdikadirova(D), Kymbat Zhienbayeva(D), Bahyt Suleimenova(D, Yuliay Talaspekova*iD, Asima Karipova (i), Irina Baryshnikova (D), Murat Zhalmakhanov (D), Lubov Piven (D), Irina Medvedeva(i), Sergey ZhuravleviD, Nazgul Omarbekova iD

Department of Pathology, Non-profit Joint-Stock Company “Karaganda Medical University”, Karaganda, Kazakhstan

Bon: Ksenija Bogoeva-Kostovsk Citation: Zhautikova S, Abdikadirova K, Zhienbayeva K Suleimenova B, Pavlovna TY, Karipova A, Baryshnikova Zhalmakhanov M, Piven L, Medvedeva I, Zhuravlev S, of Metabolic and Morphofunctional Disorders of Thyroid
onarbekovation and Adrenal Glands in Diabetes Mellitus and Obesity. OpenAccess Maced J Med Sci. 2022 Feb 03; 10(B):232-239 https://doi.org/10.3889/oamjms.2022.815 Keywords: Diabetes mellitus of type 1 and 2; Ischemic heart disease; Obesity; Thyroid gland; Adrenal glands; Biochemical disorders; Hormonal status; Morphology "Correspondence: Yuliay Talaspekova, Departmen of Pathology, Non - Profit Joint - Stock Company,
"Karaganda Medical University." Gogol str. 40, Karaganda 1000008, Kazakhstan. E-mail: talaspekovaula Received: 02-Dec-2021 Revised: 20-Jan-2022 Accepted: 24-Jan-2022 Copyright: $\odot 2022$ Saule Zhautikova, Khamida Abdikadirova, Kymbat Zhienbayeva, Bahyt Suleimenova, Yuliay Talaspekova, Asima Karipova, Irina Baryshnikova, Murat Zhalmakhanov, Lubov Piven, Irina Medvedeva, Sergey Zhuravlev, Nazgul Omarbekova Funding: This research did not receive any financia Competing Interests: The authors have declared that no competing interests Open Access: This is an open-access article distributed under the terms of the Creative Commons Attribution-

\section{Abstract}

BACKGROUND: The issues of the pathogenetic relationship of thyroid-adrenal disorders in diabetes mellitus (DM) remain relevant, despite certain advances in the study of the pathogenesis and the clinic of DM. These issues are especially actual in the case of a combination of DM with ischemic heart disease (IHD) and obesity.

AIM: The aim was to reveal the pathogenetic mechanisms of the relationship between metabolic and morphofunctional thyroid-adrenal disorders in DM and obesity.

METHODS: The study included 395 patients with type 1 and 2 diabetes. The diagnosis of DM was verified in accordance with International Programs and was based on WHO criteria. The glycemic level of patients was determined using a One Touch $\AA$ basic glucometer (Johnson\&Johnson, USA). The degree of carbohydrate metabolism compensation was assessed by the level of glycated hemoglobin ( $\mathrm{HbA1c}$ ), determined using a laboratory analyzer DCA-2000 MT (BAYER, Germany). The concentration of C-peptide in the blood serum was determined by the method of immunoluminometric analysis "Immunotech" (Czech Republic). Caro and HOMA-IR indices were calculated to identify and assess the insulin resistance (IR). The indices of hormone metabolism were determined by ELISA using DSL kits (USA) with subsequent measurement of optical density on a Spectra Classic reader from Tecan (Austria): Corticotropic hormone, adrenaline, noradrenaline, cortisol, free hydrocortisone; 17-ketosteroids, 17-oxycorticosteroids, glucogone, insulin, somatotropic hormone (STH); thyroid-stimulating hormone (TSH) thyroxine $\left(\mathrm{T}_{4}\right)$; and thyroxine $\left(\mathrm{T}_{3}\right)$. Instrumental-functional and radiation research methods. Ultrasound examination of the adrenal glands, thyroid gland, lungs, liver, and kidneys was performed in all patients. Morphological changes were assessed using histological and morphometric methods.

RESULTS: Disorders of carbohydrate metabolism in diabetic patients were revealed by increased glycemic parameters - in 2.14 times, immunoreactive insulin (IRI) - in 2.8 times, HbA1c - in 1.85 times, and HOMA - in 5.3 times compared with the control group. The following indicators were significantly higher in patients with combination of DM, IHD, and obesity: Glycemia - in 2.29 times $(p<0.05)$, IRI - in 3.81 times, HbA1c - in 2.01 times, and HOMA - in 7.76 times compared with Co and CIHD groups. An increase in the content of pyruvate and lactate and the ratio in the DM2O and DM2IHDo groups indicate an acceleration of glycolysis and the degree of pyridine nucleotides reduction, as well as excessive lipolysis and progression of tissue hypoxia. Thus, the rate of glucose oxidation in patients of DM2o subgroup is reduced in 3.02 times, in patients of DM2IHDo subgroup $(p<0.05)-$ in 3.18 times compared with Co group. Computed tomography (CT) revealed an increase in the volume of adipose tissue in relation to muscle and bone tissue in patients of DM2O and DM2IHDo subgroups. Abdominal obesity type is expressed in these patients. An increase of glucose promotes its conversion into triglycerides (TG) of adipose tissue under the condition of hyperinsulinemia. Lipogenesis in the body of patients with DM and coronary heart disease increases, and obesity develops. The increase of cortisol and TSH $(p<0.01)$ levels was observed in patients of DM2o, DM2IHDo groups compared with the control groups. In patients of DM1 group, the parameters of norepinephrine were increased in 2.23 times, TSH $(p<0.01)-$ in 3.15 times, and the content of STH was reduced in 3.76 times and the content of cortisol - in 1.5 times significantly $(p<0.01)$ compared to $C 1$ group. Ultrasound and CT with contras revealed diffuse adrenal hyperplasia, signs of a decrease in the size of the thyroid gland with a medium-grained structure, areas of its cystic degeneration in the form of hypoechoic zones with a medium-grained echo structure. Thus, the expressed atrophic processes in the lobes of the thyroid gland were observed in $27(15.4 \%)$ patients of DM2 group and in $13(13 \%)$ patients of DM2CHDo group. Analysis of spectral characteristics during Dopple ultrasonography of the thyroid gland vessels made it possible to determine low peak systolic blood flow velocities in $\mathrm{CIHD}$ group. Pathomorphological examination of the adrenal glands on electronograms recorded that the porosity of the walls of the sinusoidal capillaries increased primarily within the bundle zone of the cortex. Corticocytes of the fascicular and reticular zones underwent degenerative and necrotic changes. Along with this, some of the epithelia cells contained the usual number of light and an increased number of dark fat droplets. The study of the sectiona material revealed signs of suppression of the function of the thyroid gland, dystrophic changes in the cells increased which contributed to a decrease in the functional capabilities of the follicular epithelium, destruction of individual thyrocytes, and substitutional sclerosis with an increase in the exchange surface area in the blood-tissue barriers. 
CONCLUSION: The main pathogenetic mechanism of suppression of the structural and functional state of the adrenal and thyroid glands is due to dystrophic changes in the microvasculature. With the addition of ischemic heart disease, the oppression of their functions of the glands develops, which leads to destruction, an increase in vascularization, porosity of the capillary walls, and tissue hardening. There is a further aggravation of hormonal and metabolic disorders in patients with DM with coronary heart disease. It is confirmed by a significant increase in the lactate/pyruvate index and the index of IR. The volume of adipose tissue in relation to muscle and bone tissue on CT was significantly higher $(p<0.05)$ compared to the control groups. The predominance of the abdominal type of fat deposition is expressed.

\section{Introduction}

Diabetes mellitus (DM) is one of the pressing health problems in all countries. According to the WHO, there are currently 285 million patients with diabetes in the world, and by 2025 their number will be 380 million, and by $2030-435$ million [1]. At the same time, the real rate of increase in the incidence is significantly ahead of even such depressing forecasts of statisticians. The main causes of death in patients with diabetes are cardiovascular and cerebrovascular diseases [2]. There are significantly more manifestations of heart failure after myocardial infarction in the presence of relatively well-preserved systolic heart function in patients with diabetes [3].

The pathogenetic relationship of thyroidadrenal disorders in diabetes remains poorly understood, despite certain advances in the study of the pathogenesis, clinical picture, and treatment of diabetes, especially when combined with coronary heart disease (ischemic heart disease [IHD]) and obesity. However, the results of the influence of metabolic control, the presence and severity of complications of diabetes require clarification. In this regard, the study of the relationship between the frequency, structure, and severity of thyroid and adrenal dysfunctions is of undoubted interest.

The aim of the research was to reveal the pathogenetic mechanisms of the relationship between metabolic and morphofunctional thyroid-adrenal disorders in DM.

\section{Materials and Methods}

395 patients with DM type 1 and 2 were monitored. The diagnosis of DM was verified in accordance with International Programs and was based on WHO criteria. The age of patients with DM type 1 (DM1) and the control group C1 (practically healthy persons) averaged $37.5 \pm 1.68$ years. The duration of DM1 ranged from 5 to 22 years with an average of $11.43 \pm 0.32$ years. The average age of patients with DM type 2 (DM2) and the control group C2 was $53.74 \pm 2.48$ years. The average duration of the disease in patients with DM2 was $9.2 \pm 0.61$ years. The age of patients with DM with IHD, the comparison groups KIHD (without DM2) and Co (with obesity) averaged $60.1 \pm 2.98$ years. The duration of diabetes from 6 to 10 years was recorded in $35(12.72 \%)$ patients, from 10 to 15 years - in $153(55.63 \%)$ patients, more than 15 years - in $87(31.63 \%)$ patients. In the studied patients, the Rose [4], [5], questionnaire recommended by the WHO for the detection of exertional angina, anamnestic data on hospitalization with angina pectoris or myocardial in fact, data of daily monitoring of ECG, and EchoCG was used for screening. The average duration of IHD in patients was $10.7 \pm 1.47$ years. Exertional angina pectoris of functional Class II was registered in 50 patients $(50.0 \%)$, exertional angina pectoris of functional Class III - in $23 \%$ of patients, and exertional angina pectoris of functional Class IV - in $9.0 \%$ of patients. $18(18.0 \%)$ patients had a history of myocardial infarction. Patients of groups DM2 with IHD received antianginal therapy and beta-blockers.

According to the criteria of the International Diabetes Federation (IDF) (2005) [6],[7] abdominal obesity is diagnosed with a waist volume of more than $94 \mathrm{~cm}$ in European men and more than $80 \mathrm{~cm}$ in women. The body mass index (BMI) was calculated according to the WHO body mass classification (1997). The glycemic level in patients was determined using a One Touch ${ }^{\circledR}$ basic glucometer (Johnson\&Johnson, USA). The degree of carbohydrate metabolism compensation was assessed by the level of glycated hemoglobin (HbA1c), determined using a laboratory analyzer DCA-2000 MT (BAYER, Germany). The concentration of C-peptide in the blood serum was determined by the method of immunoluminometric analysis "Immunotech" (Czech Republic). Caro and HOMA-IR indices were calculated to identify and assess the insulin resistance (IR).

A complex of laboratory-biochemical methods for lipid and protein metabolism by enzymatic colorimetric methods (CHOD-PAP, GPO-PAP) on an automatic biochemical analyzer "TARGA-2000" ("BIOTECHNICA INSTRUMENT," Italy) was used. Blood serum enzymes were determined (according to Reitman and Frenkel) to assess the functional state of internal organs aspartate aminotransferase, alanine aminotransferase, and lactate dehydrogenase [8],[9]. The indices of hormone metabolism were determined by ELISA using DSL kits (USA) with subsequent measurement of optical density on a Spectra Classic reader from Tecan (Austria): Corticotropic hormone, adrenaline, noradrenaline, cortisol, free hydrocortisone; 17-ketosteroids, 17-oxycorticosteroids, glucogone, insulin, somatotropic hormone (STH); thyroid-stimulating hormone (TSH); thyroxine $\left(\mathrm{T}_{4}\right)$; and thyroxine $\left(\mathrm{T}_{3}\right)$. 


\section{Instrumental-functional and radiation research methods}

Ultrasound examination of the adrenal glands, thyroid gland, lungs, liver, and kidneys was performed in all patients.

The vascularization of the thyroid gland, the presence of masses was assessed by color mapping, and the type of blood supply was also assessed. A spectrometric study of exhaled air was carried out after taking a test dose of glucose enriched in the ${ }^{13} \mathrm{C}$ isotope. A non-invasive diagnostic method based on determining of the rate of glucose oxidation and its Doppler intermediate metabolites (pyruvate and lactate) before and after glucose load was used to assess glucose metabolism.

\section{Electron microscopic studies}

Material for morphological examination and autopsy material was stained with hematoxylin and eosin, iron hematoxylin and Masson's method, the sections were examined in an Opton light microscope and photographed [10],[11].

\section{Morphometric studies}

The volumetric ratios of tissue components were determined using an ocular measuring grid for cytohisto stereometric studies of Avtandilov [12]. The activity of the studied enzymes was determined cytophotometrically by the optical density of cell areas on an MBR-15 microscope with an photometric fluorescent nozzles-1 attachment [13],[14],[15].

The obtained data were processed by the method of variation statistics. The arithmetic mean sample $(\mathrm{M})$, standard deviation $(\delta)$, and arithmetic mean error $(\mathrm{m})$ were determined [16]. The significance of the differences was assessed by the Student's test. The relationship of quantitative traits was studied by the method of correlation analysis. The significance of the linear correlation coefficient (Pearson) and the rank coefficient (Spearman) was checked on the basis of the Student's t-test [17]. To quantify the tightness of the relationship, the correlation coefficient $r$ was used, which is calculated in Excel using the $f_{x}$ function, then statistical functions, the CORREL function.

\section{Results}

Considering the importance of BMI for patients with diabetes, subgroups of DM2n were identified - $36(20.6 \%)$ patients with normal BMI and a subgroup of DM2o - $139(79.4 \%)$ patients with obesity (BMI 35-39.9 kg/m²), DM2IHDn with normal BMI $24(24.0 \%)$ patients and DM2IHDo - $76(76.0 \%)$ patients with obesity. Table 1 shows the average values of BMI, waist circumference (WC), and WC/hip circumference (WC/HC).

The BMI indicator in DM2o subgroup in men was significantly $(p<0.01) 1.68$ times higher and in women $(p<0.5) 1.61$ times higher than in the C2 group. The most significantly $(p<0.01)$ high BMI was in the DM2IHDo subgroup in men (1.73 times more compared to $\mathrm{C} 2$ and 1.62 times more than $\mathrm{CIHD}$ ), and in 1.71 times higher in women $(p<0.5)$ compared with women $\mathrm{C} 2$ and in 1.59 times higher compared with CIHD. The nature of the distribution of the fat layer was determined by WC (Table 1). Thus, an abdominal type of obesity was revealed in patients of DM20, DM2IHDo groups, which is one of the important clinical signs of IR.

Disorders of carbohydrate metabolism in diabetic patients were detected by increased indicators of glycemia, immunoreactive insulin (IRI), HbA1c, C-peptide, Caro and HOMA indices, lactate, pyruvic acid (PVA), and lactate/PVA ratio [18]. The following indices were significantly $(p<0.05)$ higher in patients with DM with obesity: Glycemic level - in 2.14 times, IRI - in 2.8 times, HbA1c - in 1.85 times, and HOMA - in 5.3 times compared with Co group. The following indicators were significantly higher in patients with combination of DM, IHD and obesity: Glycemia - in 2.29 times $(p<0.05)$, IRI - in 3.81 times, HbA1c - in 2.01 times, and HOMA - in 7.76 times compared with Co and CIHD groups (Figure 1).

An increase in the content of pyruvate, lactate and their ratio in patients of DM2o and DM2IHDo groups indicates an acceleration of glycolysis and the degree of pyridine nucleotide recovery, as well as excessive lipolysis and progression of tissue hypoxia. The rate of glucose oxidation in the CIHD group decreased to 7.20 and 5.50, respectively, at the background of glucose load with isotopes when taking $10.0 \mathrm{~g}$ and $20.0 \mathrm{~g}$ of glucose. The rate of glucose oxidation decreased to 4.74 and 3.97 respectively in patients with $\mathrm{DM} 2 \mathrm{n}$ and $\mathrm{DM} 2 \mathrm{o}$. The lower results were recorded in patients of DM2IHDn and

Table 1: Average values of BMI, WC in patients with diabetes mellitus

\begin{tabular}{|c|c|c|c|c|c|c|c|c|c|}
\hline \multirow[t]{3}{*}{ Index } & \multicolumn{5}{|c|}{ Groups of patients with DM } & \multicolumn{2}{|c|}{ Control groups } & \multicolumn{2}{|c|}{ Comparison groups } \\
\hline & \multirow[t]{2}{*}{ DM1 $(n=120)$} & \multicolumn{2}{|l|}{ DM2 $(n=175)$} & \multicolumn{2}{|l|}{ DM2IHD $(n=100)$} & \multirow[t]{2}{*}{ C1 $(n=20)$} & \multirow[t]{2}{*}{$C 2(n=20)$} & \multirow[t]{2}{*}{$\mathrm{CIHD}(\mathrm{n}=20)$} & \multirow[t]{2}{*}{ Co $(n=20)$} \\
\hline & & DM2n $(n=36)$ & DM2o $(n=139)$ & DM2IHDn $(n=24)$ & DM2IHDo $(n=76)$ & & & & \\
\hline BMI, $\mathrm{kg} / \mathrm{m}^{2}-\mathrm{men}$ & $23.06 \pm 1.1^{*}$ & $24.85 \pm 1.13$ & $38.88 \pm 1.58^{\star *}$ & $25.02 \pm 1.23$ & $39.93 \pm 1.23^{\star *}$ & $22.75 \pm 0.2$ & $23.06 \pm 0.21$ & $24.52 \pm 0.2$ & $38.26 \pm 0.26$ \\
\hline - women & $22.52 \pm 1.05$ & $24.98 \pm 1.12$ & $37.46 \pm 1.54^{*}$ & $24.84 \pm 1.22$ & $39.75 \pm 1.22^{*}$ & $22.26 \pm 0.2$ & $23.16 \pm 0.21$ & $24.94 \pm 0.2$ & $37.88 \pm 0.25$ \\
\hline WC, $\mathrm{cm}$ - men & $76.11 \pm 3.7$ & $77.1 \pm 4.17$ & $105.6 \pm 5.7^{\star \star}$ & $85.4 \pm 4.1$ & $106.03 \pm 0.94^{\star \star}$ & $80.52 \pm 0.72$ & $83.4 \pm 0.75$ & $82.2 \pm 0.74$ & $104.6 \pm 0.94$ \\
\hline - women & $75.08 \pm 3.8$ & $78.1 \pm 3.78$ & $104.2 \pm 5.11^{* *}$ & $78.3 \pm 3.84$ & $105.32 \pm 0.94^{\star *}$ & $77.8 \pm 0.7$ & $77.39 \pm 0.7$ & $76.4 \pm 0.69$ & $104.2 \pm 0.98$ \\
\hline WC/HC- men & $0.83 \pm 0.04$ & $0.85 \pm 0.04$ & $0.94 \pm 0.05$ & $0.86 \pm 0.04$ & $0.96 \pm 0.01$ & $0.83 \pm 0.01$ & $0.80 \pm 0.01$ & $0.82 \pm 0.01$ & $0.94 \pm 0.01$ \\
\hline - women & $0.80 \pm 0.03$ & $0.81 \pm 0.04$ & $0.91 \pm 0.04$ & $0.82 \pm 0.04$ & $0.92 \pm 0.01$ & $0.80 \pm 0.01$ & $0.79 \pm 0.01$ & $0.78 \pm 0.01$ & $0.90 \pm 0.01$ \\
\hline
\end{tabular}




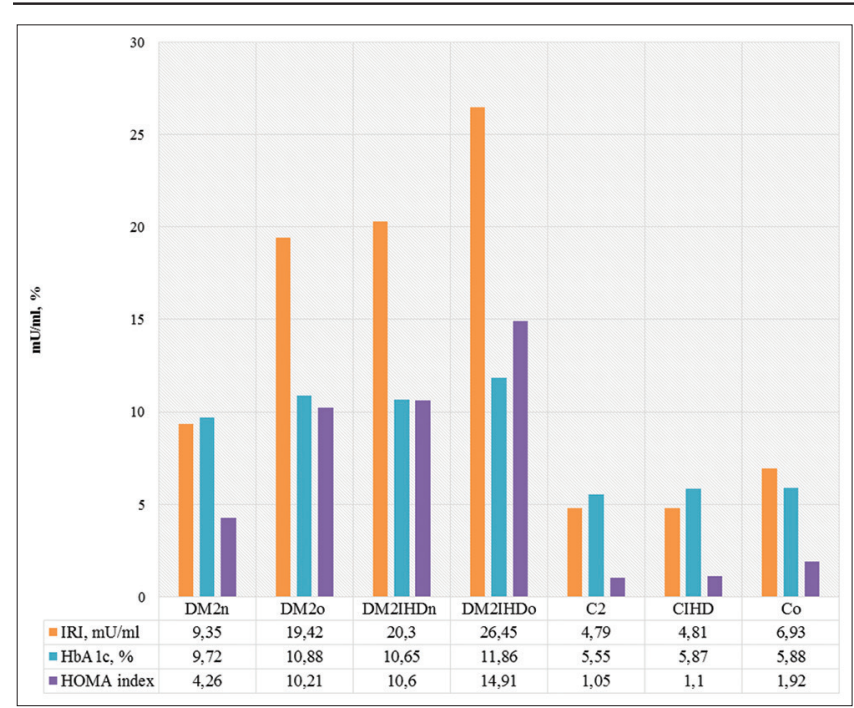

Figure 1: Indicators of immunoreactive insulin, glycated hemoglobin and HOMA index in the blood of patients with DM

DM2IHDo -4.64 and 3.64. Thus, the rate of glucose oxidation in patients of DM2o subgroup was lower in 3.02 times, and in patients of DM2IHDo subgroup ( $p<$ 0.05 ) - in 3.18 times lower than in the Co group.

Lipid metabolism in patients with DM1 was manifested by impaired lipogenesis and lipolysis, which was expressed in a significant $(p<0.01)$ increase of TGs level - in 1.33 times, atherogenic index (AI) - in 1.88 times, and total cholesterol (TC) - in 1.92 times. The level of TGs and atherogenic index $(p<0.001)$ in patients with DM2o increased in 1.8 times, levels of free fatty acids (FFA), low-density lipoprotein (LDL), very low-density lipoprotein (VLDL), and $\mathrm{apoB}$ - in 2 times [19],[20],[21],[22]. In DM2IHDo patients, expressed disorders were manifested in a significant $(p<0.001)$ increase in the level of TGs in 1.96 times and atherogenic index in 2 times (Figure 2). Glucose level increase promotes its conversion into TG of adipose tissue under the condition of hyperinsulinemia, lipogenesis increases in the body of patients of DM2IHD group, and obesity develops.

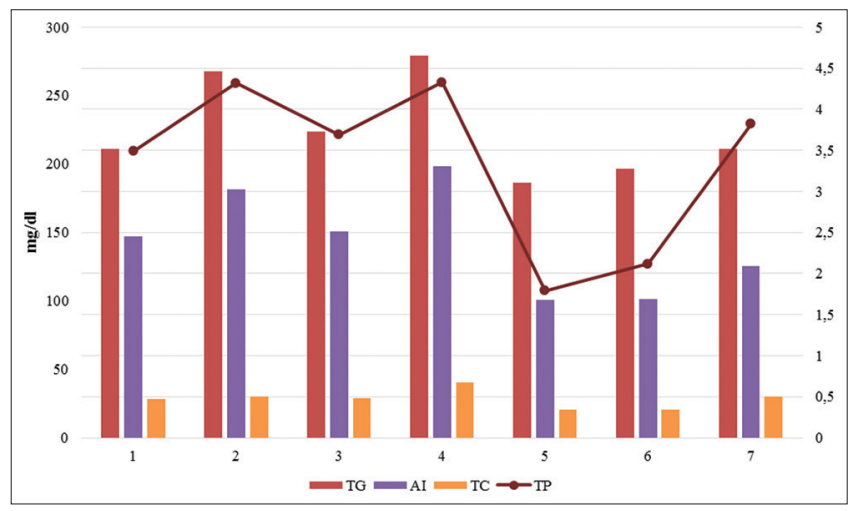

Figure 2: Indicators of lipid and protein metabolism in blood in patients with $D M$

An insignificant decrease in total protein (by $3.1 \mathrm{mmol} / \mathrm{L}$ ) and in the albumin fraction (by $1.8-2.2$ $\mathrm{mmol} / \mathrm{L}$ ) was found in patients with DM2. Moreover, these indicators decreased by 1.15 times in DM1 compared with $\mathrm{C} 1$ group, which was associated with the presence of diabetic nephropathy in them.

Comparing the results of the hormonal status in patients with DM2o, DM2IHDo with abdominal obesity, there was an increase in the indices of norepinephrine, cortisol, TSH $(p<0.01)$ and a significant $(p<0.01)$ in 4.17 time decrease in the content of STH in comparison with the control groups (Figure 3). However, in patients with DM1, the parameters of norepinephrine were increased in 2.23 times, TSH $(p<0.01)-$ in 3.15 times. STH content was significantly $(p<0.01)$ reduced in 3.76 times, cortisol - in 1.5 times compared with $\mathrm{C} 1$ group.

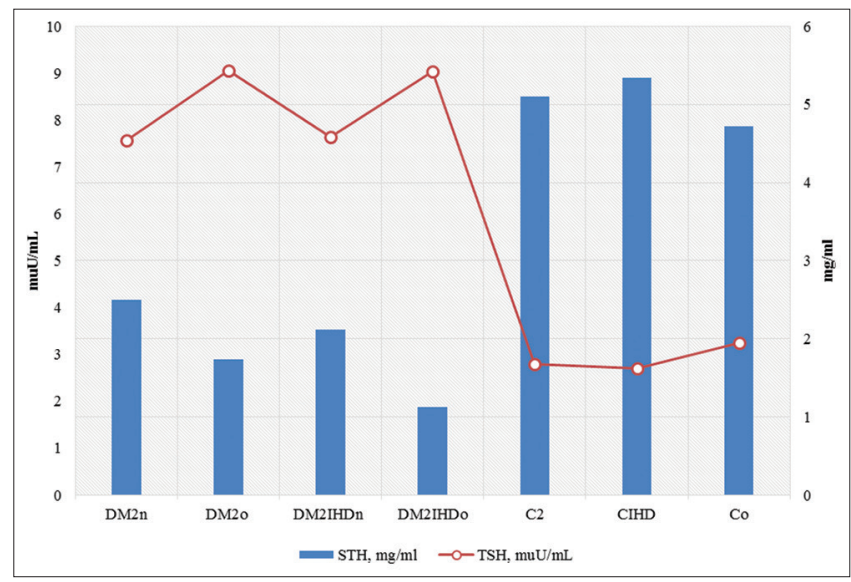

Figure 3: Indicators of somatotropic hormone and thyroid stimulating hormone hormones in the blood in patients with DM

A biopsy of $5 \mathrm{~mm}^{3}$ of the subcutaneous tissue of the abdomen was performed in all groups of patients In order to determine the morphofunctional state of adipose tissue. Morphologically, hyperplasia and hypertrophy of fat cells were recorded in adipose tissue of patients of Co, DM2o, DM2IHDo groups. At the same time, their volume increased in 5-7 times compared with DM2 group. When examining $1 \mathrm{~mm}^{3}$ of adipose tissue of patients of DM2o, DM2IHDo groups, $30 \%$ of glycogen content was revealed, while group Co did not have glycogen. Thus, fat cells are able to synthesize glycogen from glucose. The presence of glycogen in patients with DM2 and obesity indicates that it is converted to fat and deposited in the depot [23].

The ratio of adipose tissue to muscle and bone in groups Co, DM2O, and DM2IHDo with obesity of 2-3 degrees is shown on computer tomography (Figure 4).

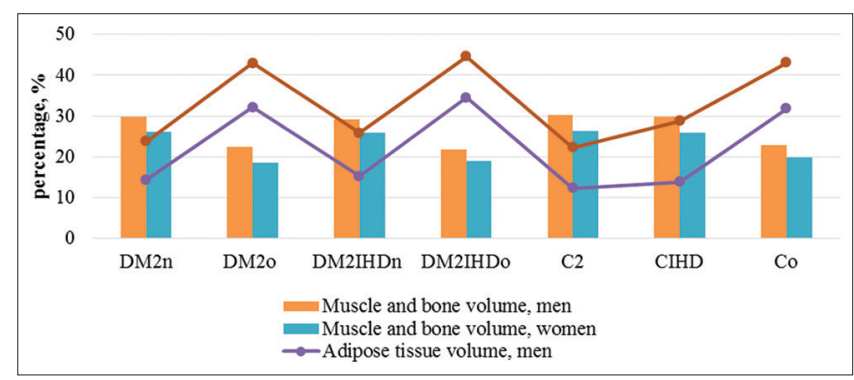

Figure 4: Distribution of muscle, bone, adipose tissue in men and women with DM 
Thus, the volume of adipose tissue was in 1.92 times higher in patients of DM2o group compared with the $\mathrm{C} 2$ group. It was in 2.0 times higher in patients of DM2IHDo group than in C2 and in 1.54 times higher than in patients of CIHD groups. Thus, the presence of abdominal type of fat deposition and its volume in the body do not depend on the age and gender of patients with diabetes.

Ultrasound and computed tomography (CT) scan with contrast revealed diffuse adrenal hyperplasia in $2(1.6 \%)$ patients with DM1, an increase in the size of the adrenal glands in 21 (15.1\%) patients of DM2o group, and in $13(17.1 \%)$ patients of DM2IHDo group. Signs of a decrease in the size of the thyroid gland with a mediumgrained structure were found in $17(9.7 \%)$ patients of DM2o group. Examination of the thyroid gland in $3(2.5 \%)$ patients of DM1 and in $1(0.57 \%)$ patient of DM2n group revealed areas of cystic degeneration in the form of hypoechoic zones with a medium-grained echo structure. A decrease in the size of the thyroid gland on CT scan was confirmed by ultrasound. Thus, expressed atrophic processes in the lobes of the thyroid gland were observed in $27(15.4 \%)$ patients with DM2 and in 13 (13\%) patients with DM2IHDo.

Pathomorphological examination of the adrenal glands on electronograms indicated an increase in erythrocyte sludge in microvessels. The porosity of the walls of sinusoidal capillaries increased primarily within the bundle zone of the cortex (Figure 5a). Expressed diapedesis of erythrocytes into the interstitial space was found. Corticocytes of the fascicular and reticular zones underwent degenerative and necrotic changes. Sinusoidal capillaries were sharply dilated, often contained sludge erythrocytes; their cytoplasm contained a small amount of small fat droplets. Along with this, some of the epithelial cells contained the usual number of light and an increased number of dark fat droplets (Figure 5b).

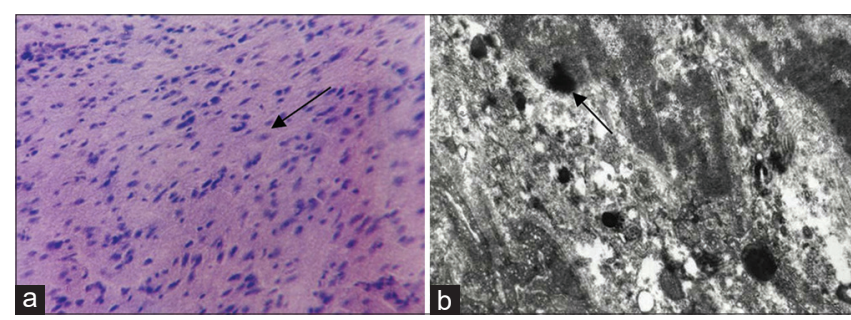

Figure 5: ( $a$ and b) Pathomorphology of adrenals in patients with DM

Thus, the volume of adipose tissue was in 1.92 times higher in patients of DM2o group compared with the $\mathrm{C} 2$ group. It was in 2.0 times higher in patients of DM2IHDo group than in C2 and in 1.54 times higher than in patients of CIHD groups. Thus, the presence of abdominal type of fat deposition and its volume in the body do not depend on the age and gender of patients with diabetes.

Ultrasound and CT scan with contrast revealed diffuse adrenal hyperplasia in $2(1.6 \%)$ patients with DM1, an increase in the size of the adrenal glands in $21(15.1 \%)$ patients of DM2o group and in $13(17.1 \%)$ patients of DM2IHDo group. Signs of a decrease in the size of the thyroid gland with a medium-grained structure were found in $17(9.7 \%)$ patients of DM2o group. Examination of the thyroid gland in $3(2.5 \%)$ patients of DM1 and in $1(0.57 \%)$ patient of DM2n group revealed areas of cystic degeneration in the form of hypoechoic zones with a medium-grained echo structure. A decrease in the size of the thyroid gland on CT scan was confirmed by ultrasound. Thus, expressed atrophic processes in the lobes of the thyroid gland were observed in 27 (15.4\%) patients with DM2 and in $13(13 \%)$ patients with DM2IHDo.

Dystrophic changes in the microcirculatory bed lead to a decrease in the functional capabilities of the follicular epithelium, destruction of individual thyrocytes, and substitutional sclerosis (Figure 6) with an increase in the exchange surface area in the blood-tissue barriers.

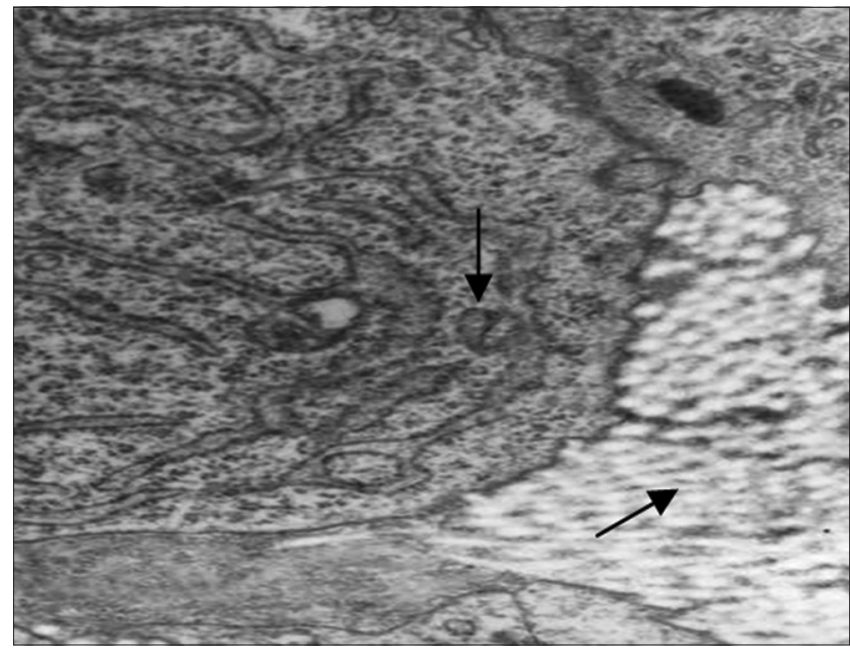

Figure 6: Pathomorphology of the thyroid gland of a patient with T2DM. Single secretory granules (SG). Destruction of individual thyrocytes, replacement sclerosis (RS). SW. x 12800

\section{Discussion}

In accordance with the criteria of the IDF (2005), abdominal obesity is diagnosed with WC more than $94 \mathrm{~cm}$ in European men and more than $80 \mathrm{~cm}$ in women. Thus, the study of the distribution of the fat layer by the WC in the control groups $\mathrm{C} 1, \mathrm{C} 2$, and CIHD showed this index from $80.52 \mathrm{~cm}$ to $82.2 \mathrm{~cm}$ in men and from $76.8 \mathrm{~cm}$ to $77.39 \mathrm{~cm}$ in women. WC index in patients of DM1, DM2n, and DM2IHDn groups with normal BMI did not differ significantly from the control groups. Whereas this indicator in groups with obesity was more than $100 \mathrm{~cm}$ : In men of the Co group $-104.6 \mathrm{~cm}$ and in women $-104.2 \mathrm{~cm}$. WC in the DM2o subgroup was $105.6 \mathrm{~cm}$ in men and $104.2 \mathrm{~cm}$ in women. WC index was higher by $1.0 \mathrm{~cm}$ in DM2IHDo subgroup (106.03 cm and $105.32 \mathrm{~cm}$, respectively), which indicates an abdominal fat distribution. 
The data from studies of the relationship between waist and hip measurements in healthy individuals and in patients with DM are very similar. Henec, the highest indicator was also in the Co group and in the DM2o and DM2IHDo subgroups: in men - 0.94, 0.94, and 0.96; in women $-0.90,0.91$, and 0.92 . The $\mathrm{WC} / \mathrm{HC}$ index in the control groups and patients with DM1 and DM2n with normal BMI was in the range of $0.80-0.86$ in men and $0.78-0.81$ in women. Thus, the abdominal type of obesity was revealed in patients with DM20 and DM2IHDo groups, which is an important clinical sign of IR.

Violation of the carbohydrate balance determines the violation of energy metabolism and the functional state of many organs and systems. When comparing the indices of IRI and C-peptide, the lowest indices were found in the group of patients with CD1 - in 4.25 times $(p<0.01)$ less than in the C1 group. Significantly ( $p<0.001$ ), the highest rates were recorded in patients with abdominal obesity in DM2o group (in 4.05 times compared to C2 group and in 2.8 times compared to Co group) and DM2IHDo group (in 5.49 times compared to CIHD group and in 3.81 times compared to Co subgroup).

If patients with DM2 with a very similar clinical picture of the disease and the same duration of the disease have different insulin content, then this is due to the presence of obesity. Additionally, IR was calculated using Caro and HOMA indices. The Caro index less than 1.0 was determined in the subgroups DM2o, DM2IHDn, DM2IHDo, and Co. IR according to the HOMA index (if the indicator was more than 2.77 ) significantly differed $(p<0.01)$ in patients with DM2o, DM2IHDn, and especially DM2IHDo. Hyperglycemia is the starting point of many mechanisms that contribute to the development of complications of diabetes. $\mathrm{HbA} 1 \mathrm{c}$ has a direct correlation with the level of glucose in the blood and it is an integral indicator of the compensation of carbohydrate metabolism for a long time (60-90 days). Significantly ( $p<0.001)$ the highest indicator $(12.03 \%)$ was in patients with type DM1 (2.52 times more than in $\mathrm{C} 1$ group). $\mathrm{HbA} 1 \mathrm{c}$ averaged $9.72 \%$ in patients of DM2n subgroup. HbA1c significantly $(p<0.01)$ increased in DM2o subgroup 1.96 times compared with $\mathrm{C} 2$ group and in 1.85 times $(p<0.01)$ compared to Co group.

Greatimportanceinthemechanismofdisturbances in the processes of glycolysis and gluconeogenesis in DM is attached to the change in the functional state of the pyruvate dehydrogenase system of mitochondria, which is involved in the oxidative decarboxylation of pyruvate to acetyl-CoA. When determining the content of pyruvate and lactate in the blood, as well as their ratio - the lactate/ pyruvate index, we can judge the accelerated glycolysis, the degree of pyridine nucleotide reduction, as well as excessive lipolysis and the progression of tissue hypoxia. Thus, in patients with DM2 in subgroups with obesity (DM2o and DM2IHDo), the content of lactate and PVA in blood was increased. At the same time, an increase in the lactate/pyruvate index up to $0.029-0.030$ was noted in these patients, which confirms the development of tissue hypoxia. It is possible that this effect is realized through a decrease in IR in patients with DM2, and the severity of IR depends on the state of the pyruvate dehydrogenase complex [24].

Wehaveproved thattheprocessesoflipogenesis and lipolysis are impaired in patients with DM1, which was expressed in a significant $(p<0.01)$ increase of TG level in 1.33 times and the atherogenic index - in 1.88 times; an increase of FFA in 1.92 times [25], [26]. In patients with DM2, the spectrum of dyslipidemic disorders expands: A significant increase of TG $(p<0.01)$ in 1.8 times and $\mathrm{Al}(p<0.001)$ in 2.03 times, FFA, LDL, VLDL, and apoB. When comparing patients with DM2IHD, we revealed the expressed disorders in obese patients, which was manifested in a significant ( $p<0.001$ ) increase of TG levels (in 1.96 times) and an atherogenic index (in 2.04 times) [27], [28]. An increase of glucose level promotes the conversion of adipose tissue into TGs under the condition of hyperinsulinemia, while lipogenesis in the body of patients with DM2IHD increases and obesity develops [29].

Particular importance in DM2 pathogenesis is attributed to the counter insular hormone cortisol, which affects the sensitivity of cell receptors [30], [31]. The level of cortisol was шт 1.5 times lower in patients with DM1, especially in patients with underweight $\left(\mathrm{BMI}<18.5 \mathrm{~kg} / \mathrm{m}^{2}\right)$. The cortisol index was in 3.2 times higher in the DM2n subgroup $(p<0.05)$ compared with C2 group and in 2.32 times higher in DM2o subgroup compared with $\mathrm{C} 2$ group. The cortisol index was more than one in patients with IHD, and was $1.18 \mathrm{~nm} /$ day in CIHD group ( $p<0.01$ ), $1.58 \mathrm{~nm} /$ day in DM2IHDn group, and $1.66 \mathrm{~nm} /$ day in DM2IHDo group. It indicates an increase in the activity of the fascicular zone of the adrenal cortex at IHD. The glucagon index was within the normal range, but in all patients with DM2 it was in 1.77 times higher $(p<0.01)$ than in $C 2$ group.

A study of the content of thyroid hormones $\mathrm{TSH}, \mathrm{T}_{3}$, and $\mathrm{T}_{4}$ in blood of patients with $\mathrm{DM}$ and control groups revealed a significant $(p<0.05)$ increase of TSH in 3.23 times in DM2o compared to C2 group and in 2.78 times compared with Co group. TSH index was significantly $(p<0.01)$ increased in 2.34 times in patients of DM2IHDo group compared with CIHD group and in 2.77 times compared with Co group. When comparing the indices of $\mathrm{T}_{3}$ and $\mathrm{T}_{4}$ hormones, it was found a decrease in $7.8 \%$ in DM2 group and in $12.3 \%$ in patients of DM2IHD group. Thus, the development of primary hypothyroidism in patients of DM2O and DM2IHDo was established in terms of the level of TSH and thyroid hormones in blood.

Fat cells are able to synthesize glycogen from glucose, and the presence of glycogen in obese patients with DM2 indicates that it is converted into fat and saved in the depot [32], [33]. On CT, the percentage of adipose tissue to muscle and bone tissue in the control groups $\mathrm{C} 1, \mathrm{C} 2$, and $\mathrm{CIHD}$ in men and women varied within the physiological norm from 29.9 to 30.3. The indicators were within the same limits in DM1 and DM2 
groups, but they were reduced to $29.2 \%$ in DM2IHDn subgroup, and to $22.8 \%$ in Co group. The volume of muscle and bone tissue was reduced in 1.32 times in DM2o subgroup, and it was significantly $(p<0.05)$ decreased in 1.38 times in DM2IHDo subgroup compared to $\mathrm{C} 2$ group. The volume of adipose tissue in men in the control groups C1, C2 was $11.9 \%$ and $12.3 \%$, respectively, while there was a slight decrease to $11.6 \%$ in DM1 group. The volume of adipose tissue in patients of CIHD group was $13.8 \%$. This indicator increased in 1.10 times in patients of DM2IHDn group and in 2.5 times in DM2IHDo subgroup [34], [35], [36], [37].

In addition to ultrasound examination, the patients underwent CT with contrast enhancement, which has higher information content. CT studies were carried out in C1, C2, Co, and CIHD groups, and all patients with diabetes in dynamics. Thus, diffuse adrenal hyperplasia was revealed in $2(1.6 \%)$ patients of DM1 group, an increase in the size of the adrenal glands was detected in $21(15.1 \%)$ patients of DM2o group and in $13(17.1 \%)$ patients of DM2IHDo group.

Areas of cystic degeneration in the form of hypoechoic zones with a medium-grained echo structure were found on ultrasound of the thyroid gland in $3(2.5 \%)$ patients of DM1 group and $1(0.57 \%)$ patient of DM2n group at the age of 56 years (disease duration 11 years). Doppler ultrasonography of the thyroid gland in patients with diabetes made it possible to assess the blood flow in the posterior branch of the lower thyroid arteries and the location of the lower thyroid arteries in the peri-tracheal space. Thus, the analysis of the spectral characteristics made it possible to obtain the values of the peak systolic and diastolic velocities, as well as the angle-dependent values - the resistance index, the pulsation index, and the systolic-diastolic ratio.

Signs of suppression of the thyroid gland function were revealed when studying the sectional material. At the same time, dystrophic changes in the cells increased: The cisterns of the cytoplasmic reticulum in various cellular elements of the organ were expanded. The reaction from microvessels is characterized not only by a gradual increase in the vascularization of the follicular apparatus and, as a consequence, by an increase in the exchange surface area in the blood-tissue barriers, but also in some cases by an increase in the porosity of the capillary walls [38], [39], [40]. Dystrophic changes in the microcirculatory channel lead to a decrease in the functionality of the follicular epithelium, destruction of individual thyrocytes, and substitutional sclerosis.

\section{Conclusion}

Based on the clinical study, new scientifically grounded results were obtained: the pathogenetic mechanisms of the relationship of hormonal-metabolic thyroid-adrenal disorders in DM were revealed, which made it possible to come to the following conclusions:

1. The main pathogenetic mechanism of suppression of the structural and functional state of the adrenal and thyroid glands in $12.4 \%$ of patients with DM1 and in $15.4 \%$ of patients with DM2 is due to dystrophic changes in the microvasculature. The inhibition of their functions develops at the addition of ischemic heart disease. The peak systolic (by $22 \%$ ) and end diastolic (by $35 \%$ ) blood flow rates in the arteries of the glands decrease, which leads to destruction, an increase in vascularization, porosity of the capillary walls and tissue hardening

2. There is a further aggravation of hormonal and metabolic disorders in patients with DM with the addition of ischemic heart disease. It is confirmed by a significant increase of lactate/ pyruvate index and the IR index HOMA in 7.76 times, the decrease in the rate of glucose oxidation in 3 times, the increase of cortisol and $\mathrm{TSH}$ in 2.9 times

3. The volume of adipose tissue in relation to muscle and bone tissue on CT was significantly higher $(p<0.05)$ in 1.92 times in patients of DM2o group compared with $\mathrm{C} 2$ group, in 2.0 times higher in patients of DM2IHDo group compared with $\mathrm{C} 2$ and in 1.54 times compared with CIHD groups. Therefore, the presence of abdominal type of fat deposition and its volume in the body do not depend on the age and sex of patients with diabetes.

\section{References}

1. Seisembekov TZ, Zhautikova SB, Tuksaitova GB, Dudkina LV. Arterial Hypertension, Cortisol Excretion and 17-CS in Patients with Diabetes Mellitus. Mater. International Conference Dedicated to the $10^{\text {th }}$ Anniversary of Independence of the Republic of Kazakhstan; 2001. p. 263-5.

2. Zhautikova SB. Pathogenetic relationship of adrenal disorders and cardiovascular system in patients with diabetes mellitus. Med Ecol. 2010;4:216-9.

3. AlekseenkoSN,DrobotEV.EpidemiologyofDiabetesMellitusinthe World Latest Data. Available from: https://siestafit.ru/pro-sahar/ epidemiologiya-saharnogo-diabeta-v-mire-poslednie-dannye

4. Sokolov El. Diabetic heart. Medicine 2002;416.

5. Ragino YI, Shcherbakova LV, Denisova DV, Kuzminykh NA Yachmeneva MP, Voevoda MI. Blood lipids and angina pectoris (according to the epidemiological cardiological questionnaire of Rose) in the population of $25-45$ years of Novosibirsk. Cardiology. 2019;59(3S):30-5. https://doi.org/10.18087/cardio.2600 PMid:30990150

6. Dudinskaya EN, Machekhina LV. Insulin resistance as a link between diabetes mellitus, obesity, dyslipidemia 
and cardiovascular diseases. Effective pharmacotherapy. Endocrinology. 2021;17(5): 6-8.

7. Alimova SA, Zhuraeva KI. The use of the questionnaire method in the early diagnosis of angina pectoris as a screening test during preventive examinations of the population. Electron Sci J Biol Integr Med. 2017;6:4-9.

8. Medovshchikov VV, Eshniyazov NB, Khasanova ER, Vatsik MV, Tukhsanboev ES, Babaeva LA, et al. First identified Type 2 diabetes mellitus and prediabetes in hospitalized patients with cardiovascular diseases: frequency, compliance of baseline levels of blood pressure, lipids and $\mathrm{HbA} 1 \mathrm{c}$ with target values. Med J Clin Pharmacol Ther. 2020;4:31-5.

9. Kurashvili LV, Semechkina EA, Avdonina TS, Zueva GF, Zakharova IR. Spectrum of blood lipids in patients with insulindependent diabetes mellitus and coronary heart disease. Probl Endocrinol. 1998;44(3):1012. https://doi.org/10.14341/ probl199844310-12

10. Goncharov NP. The importance and role of hormone determination methods in the development of endocrinology as a general biological science. In: Topical Issues of Endocrinology. Russian; 2012. p. 42-9.

11. Ham A, Kormak D. Histology. Medicine. 1982;1:296.

12. Avtandilov GG. Medical morphometry. Medicine. 1990;382.

13. Pokrovsky VI. Small Medical Encyclopedia. Moscow: Medical Encyclopedia; 1996.

æ15.Sarkisov DS, Vtyurin BV. Electron microscopy of destructive and regenerative intracellular processes. Medicine. 1967;224.

16. Oivin IA. Statistical processing of experimental research results. Pathol Physiol Exp Ther. 1960;4:76-85.

17. Fletcher R, Fletcher S, Wagner E. Clinical Epidemiological Foundations of Evidence-Based Medicine. In: The Media Sphere. Vol. 345. Russian; 1998.

18. Zhautikova SB. Effectively of Using Diabetone in Treating Diabetic Cardioangiopathys. Vol. 51. $2^{\text {nd }}$ International Congress of Cadiologists of Turkish-Speaking Countries; 2000.

19. Seisembekov TZ, Zhautikova SB, Temirgalieva. Condition of Lipid Spectrum and Freely Radical Oxidation Processes by Chronic Heart Disease against a Background the Diabetes. Antalya, Turkey: $2^{\text {nd }}$ International Congress of Cadiologists of Turkish-Speaking Countries; 2000. p. 42.

20. Jangildinova $S$, Ivassenko $S$, Kelmyalene A, Yessilbayeva B, Dyussenbekova B. Determination of the product of DNA oxidation in the blood of women living in the Sub-Aral Area. Open Access Maced J Med Sci. 2019;7(10):1672-4. https://doi. org/10.3889/oamjms.2019.333

PMid:31210821

21. Okassova AK, Ilderbayev OZ, Nursafina A, Zharmakhanova GM, Rakhimova BB, Yessilbaeva BT, et al. Evaluation of lipid peroxidation under immobilization stress in irradiated animals in experiment. Open Access Maced J Med Sci. 2021;9(A):119-22. https://doi.org/10.3889/oamjms.2021.5781

22. Petrosyan AE, Diana ML, Kotova EO. Early changes in the cardiovascular system in patients with Type 1 diabetes mellitus. Med J Clin Pharmacol Ther. 2021;2:17-21.

23. Sumin AN, Bezdenezhnykh NA, Fedorova NV, Bezdenezhnykh AV, Indukaeva EV, Artamonova GV. Interrelation of visceral obesity and cardio-ankle vascular index with disorders of carbohydrate metabolism according to the study of ESSE-RF in the region of Western Siberia. Sci Pract $J$ Clin Med. 2018;96(2):137-46.

24. Volkova NI, Antonenko MI, Ganenko LA. Type 2 diabetes mellitus: A new indication for hypercorticism screening. Diagn Control Treatment. 2012;4:95-102.

25. Zhautikova SB, Seisembekov TZ, Zelenskaya VN, Bjiul EV. The characteristic of exhaled brath condensate in patients with diabetes mellitus. Eur Respir J. 2003;210.

26. Zelenskaya V, Seisembekov T, Zhautikova S, et al. Content of Total Lipids in the Exhaled Breath Condensate in Type 1 Diabetic Mellitus Patients with Different Level HbA1c. Vol. 556. $14^{\text {th }}$ ERS Annual Congress; 2004.

27. Pashentseva AV, Verbovoy AF, Galkina RA, Verbovaya N, Sharonova LA, Dolgikh YA. Management of cardiovascular risk in patients with Type 2 diabetes mellitus. Sci Pract J Clin Med. 2018;96(8):696-701.

28. Trubnikova OA, Tarasova IV, Mamontova AS, Kagan ES, Maleva OV, Barbarash OL. Predictors of moderate cognitive disorders in patients with coronary heart disease in combination with Type 2 diabetes mellitus. Sci Pract J Clin Med. 2016;94(1):31-5. PMid:27172719

29. Yuzhakova AE, Nelaeva AA, Khasanova YV. The role of adipose tissue in maintaining homeostasis of carbohydrate metabolism. Effect Pharmacother Endocrinol. 2019;1(6).

30. Kokorin SV, Kharitonova MD, Sabitova OV. Euthyroid giant goiter in a patient with cardiac rhythm and conduction disorders. Effective pharmacotherapy. Endocrinology. 2017;3:39.

31. Kan KZ, Ramachandran S, Gont K, Pushkar P. Leadership Medical education and professional development. J Graduate Med Educ. 2014;2:23-40.

32. Eshniyazov NB, Medovshchikov VV, Safarova AF, Khasanova ER, Kobalava JD. Frequency, clinical characteristics and echocardiographic phenotypes of heart failure in patients with Type 2 diabetes mellitus. Med J Clin Pharmacol Ther. 2020;4:44-8.

33. Chernomorets VS, Troitskaya EA, Kobalava JD. Orientation to central BP is a promising approach to the management of patients with uncontrolled arterial hypertension, Type 2 diabetes mellitus and chronic kidney disease. Med J Clin Pharmacol Ther. 2020;2:40-6.

34. Abikenova F, Meyramov G, Zhautikova S, Abdikadirova K, Zhienbayeva C, Talaspekova $\mathrm{Y}$, et al. Investigation of antidiabetogenic effect of the iodine-selenium concentrate in animals with chronic alloxan diabetes of varying severity. Open Access Maced J Med Sci. 2021;9(A):535-40. https://doi. org/10.3889/oamjms.2021.5873

35. Abdikadirova K, Amreyeva K, Zhautikova S, Kostyleva O, Abikenova F, Chergizova B, et al. Morphological changes in the hepatic tissue at the impact of industrial copper-bearing dust in the experiment. Open Access Maced J Med Sci. 2020;8(E):653-6. https://doi.org/10.3889/oamjms.2020.3473

36. Pavlikova EP, Sorokina AG, Potapenko AV. Prevention of Type 2 diabetes mellitus in patients with chronic heart failure. Med $J$ Clin Pharmacol Ther. 2020;1:67-74.

37. Tussupbekova M, Bakenova R, Stabayeva L, Imanbayeva G, Nygyzbayeva R, Mussabekova S, et al. Clinic morphologic and morphometric criteria for differential diagnosis of sarcoidosis and pulmonary tuberculosis. Open Access Maced J Med Sci. 2019;7(9):1480-5. https://doi.org/10.3889/oamjms.2019.315 PMid:31198459

38. Allazova SS, Novikova MS, Bobkova IN, Bobrova LA, Kotenko ON, Shilov EM. Risk factors for post-transplant diabetes mellitus in kidney recipients. Med $\mathrm{J}$ Clin Pharmacol Ther. 2019;2:44-8.

39. Troitskaya EA, Starostina ES, Kobalav JD. Prevalence of surrogate markers of atherosclerosis and arterial rigidity in patients with Type 2 diabetes mellitus and arterial hypertension. Med J Clin Pharmacol Ther. 2017;4:34-8.

40. Zvenigorodskaya LA, Mkrtumyan AM, Shinkin MV, Nilova TV, Silverstovag SY, Varvanina G, et al. Clinical significance of the key components of the adipo-cardiovascular axis in patients with Type 2 diabetes mellitus and non-alcoholic fatty liver disease Effective pharmacotherapy. Endocrinology. 17(20): 26-36. 